\title{
Aprendizaje de contenidos de Química General con uso de vídeos en carreras de Ingeniería
}

\section{General Chemistry content learning using videos in Engineering careers}

\author{
María I. Vera ${ }^{1}$, Irma I. Lucero ${ }^{2}$, Marta G. Stoppello ${ }^{3}$, Raquel H. Petris ${ }^{4}$ \\ ${ }^{1}$ Departamento de Química, Universidad Nacional del Nordeste, Argentina (marile.vera5@gmail.com) \\ ${ }^{2}$ Departamento de Física, Universidad Nacional del Nordeste, Argentina (irmaireneprof@gmail.com) \\ ${ }^{3}$ Departamento de Física, Universidad Nacional del Nordeste, Argentina (mstopello@ hotmail.com) \\ ${ }^{4}$ Departamento de Informática, Universidad Nacional del Nordeste, Argentina (raquelpetris@ @otmail.com)
}

Recibido el 20 de noviembre de 2017; revisado el 3 de abril de 2019; aceptado el 3 de abril de 2019; publicado el 1 de diciembre de 2019

\section{RESUMEN:}

Los alumnos ingresantes a carreras de Ingeniería (Eléctrica, en Electrónica y en Agrimensura) en la Facultad de Ciencias Exactas y Naturales y Agrimensura (FaCENA) de la Universidad Nacional del Nordeste (UNNE) cursan Química General de acuerdo a los planes de estudio vigentes. En este artículo se presentan los resultados de un estudio comparativo que indaga sobre el efecto de la implementación de videos educativos en la comprensión de temas centrales de la asignatura como son Nomenclatura Química, Ecuaciones Químicas y Estequiometria. Estos son los primeros temas desarrollados en clases prácticas de la asignatura, son la base para la comprensión de los demás contenidos del programa y son evaluados en el primer parcial.

Para el estudio se analizan respuestas dadas en una evaluación parcial por dos cohortes, 2014 (dictado tradicional) y 2015 (con apoyo de videos), y se aplica la prueba $\mathrm{t}$ de Student para determinar si existe diferencia significativa entre ambas.

En los tres bloques temáticos, la diferencia encontrada entre los grupos es significativa. El cálculo estadístico permite afirmar que el uso del video como recurso es la variable que está promoviendo la diferencia de tener mejores resultados en la cohorte 2015 .

La mayor diferencia entre ambos grupos se tiene para el tema estequiometria y la menor para ecuaciones químicas.
PALABRAS CLAVE: APRENDIZAJE MIXTO; TIC; VIDEO EDUCATIVO; EDUCACIÓN UNIVERSITARIA.

\begin{abstract}
:
Freshmen of Engineering degrees (Electrical, Electronics and Surveying) at the Faculty of Exact and Natural Sciences and Surveying (FaCENA) of the Universidad Nacional del Nordeste (UNNE) study General Chemistry, according to current curricula. This article presents the results of a comparative study that explores the effect of the implementation of educational videos in the understanding of fundamental contents of the subject such as chemical nomenclature, chemical equations and stoichiometry. These are the first topics developed in practical classes; they are the cornerstone of other contents of the program, and they are evaluated in the first test.

For the study, we have analysed the answers given in an evaluation by two cohorts, 2014 (traditional classroom format) and 2015 (with video support). Then, the Student's t-test has been applied to determine if there is significant difference between the two.

In the three thematic blocks, the difference found between both groups is significant. The statistical calculation allows to assert that the use of videos as a resource is the variable that is causing the difference of having better results in the 2015 cohort.
\end{abstract}


The most significant difference between the two groups is in stoichiometry, and the less significant in chemical equations.

KEYWORDS: BLENDED LEARNING; ITC; EDUCATIONAL VIDEO; UNIVERSITY EDUCATION.

\section{INTRODUCCIÓN}

\subsection{Planteamiento del problema}

La química es una disciplina que forma parte del diseño curricular de un gran número de carreras universitarias entre las que se cuentan las ingenierías ${ }^{1}$. El estudiante que ingresa a las carreras de Ingeniería Eléctrica, Ingeniería en Electrónica, Ingeniería en Agrimensura de la Facultad de Ciencias Exactas y Naturales y Agrimensura (FaCENA) de la Universidad Nacional del Nordeste (UNNE) cursa Química General en el primer cuatrimestre de acuerdo con los diseños curriculares vigentes. En las tres carreras es claro que la Química no es la asignatura conductora del perfil profesional. El número de estudiantes que la cursan es elevado; aproximadamente 350 alumnos.

El primer año de los estudios de grado juega un papel importante en la persistencia del alumnado $\mathrm{y}$, generalmente, marca el rumbo del desempeño académico de los años posteriores. De allí la importancia del acompañamiento docente y el uso de estrategias y recursos que ayuden a la mejor comprensión de los contenidos de las primeras asignaturas de la currícula. El joven que ingresa a la UNNE (y a la universidad en general) no está acostumbrado al razonamiento abstracto y al desarrollo de procedimientos del quehacer científico con justificaciones teóricas, ya que el enfoque de la enseñanza de la ciencia en la escuela secundaria se orienta más a las relaciones CTS. Dichos estudiantes provienen de distintas modalidades de educación secundaria, pudiendo no haber cursado Química. Como "nativos digitales" es común que sepan manejarse con la información y comunicación digital.

El cursado de Química General comprende clases de teoría, de resolución de problemas y experimentales de laboratorio, totalizando siete horas semanales. Las clases de teoría -tres horas

\footnotetext{
${ }^{1}$ La Resolución 1232/01 del Ministerio de Educación de la Nación (Argentina) establece que la Química debe ocupar como mínimo 50 horas de las 750 correspondientes a las denominadas Ciencias Básicas dentro de los Planes de Estudio de las carreras de Ingeniería, para cumplir con los estándares de acreditación.
}

semanales- son de grupo completo y están a cargo de la profesora titular. En las clases de resolución de ejercicios y problemas -cuatro horas semanales- los alumnos se distribuyen en grupos a cargo de jefes de trabajos prácticos con diferente formación de grado (bioquímicos, licenciados en química, ingenieros químicos).

La amplia trayectoria docente y experticia de la profesora titular y del equipo de investigación que dirige -todos docentes de primer año- les permite conocer con claridad no solo la especificidad disciplinar del contenido sino la problemática de su aprendizaje. Su experiencia les lleva a buscar nuevas estrategias superadoras de los obstáculos epistemológicos reconocidos y lo hacen en el marco del Proyecto de Investigación PI 17F001/14, "Innovación con TIC para fortalecer la enseñanza y aprendizaje de las actividades de laboratorio de Química y de Física en los primeros años de FaCENA" aprobado por la Secretaría General de Ciencia y Técnica de la UNNE (Resolución $\mathrm{N}^{\circ}$ 358/15 C.S.).

Los temas Formulación y Nomenclatura Química, Ecuaciones Químicas y Estequiometria son importantes para construir conocimiento sobre los demás contenidos del programa de la asignatura. Estos son los primeros temas desarrollados en clases prácticas de resolución de ejercicios y son evaluados en el primer examen parcial. Históricamente, los resultados obtenidos en este parcial mostraban poca comprensión de estos temas y los refuerzos para la instancia recuperadora resultaban insuficientes, de acuerdo con los resultados obtenidos. Por razones de planificación didáctica, se avanzaba en el desarrollo de los demás contenidos con la consecuencia lógica de no apropiación de los mismos.

En el año 2015 se incorporó como innovación la edición de videos que muestran la resolución de ejercicios sobre estos temas, buscando mejorar el rendimiento académico, unificar el desarrollo de los contenidos y poner a disposición del alumnado un recurso amigable que puede ser observado la cantidad de veces que necesite y en el horario que mejor se acomode a sus actividades.

\subsection{Fundamentación}

La educación no es ajena al papel de vital importancia que desempeñan las tecnologías de la información y la comunicación (TIC). Para poder dar respuesta a las transformaciones y exigencias de la sociedad -que afectan el ámbito educativo- es necesario innovar y transformar los espacios académicos, las estrategias y materiales pedagógicos (Morales Capilla, Ortiz Colón, Trujillo Torres y 
Raso Sánchez, 2015). En ese sentido, Mellado (2011) hace referencia a los profesores expertos que, conocedores de las dificultades de aprendizaje de cada tema, pueden desplegar un conjunto de estrategias para estimular y motivar el aprendizaje de sus alumnos.

Las TIC aplicadas a la enseñanza han contribuido a facilitar procesos de creación de contenidos multimedia, escenarios de teleformación y entornos colaborativos. En opinión de Medina (2009)

el diseño de medios didácticos requiere una reordenación de los clásicos y la incorporación de los digitales, pero en coherencia con el sistema de toma de decisiones, característico de la comunicación, necesitado de la fluidez que sustituya $o$ compense la interacción presencial, y la limitada bidireccionalidad de los textos escritos, ampliando la redacción de medios en la red y de uso directo. (citado en Cacheiro González, 2011, p. 70).

A los alumnos de las carreras de ingeniería de perfil no químico, cursar la asignatura Química General en el primer año de estudios no les resulta atractivo, tal vez debido a que en dicho nivel no tienen aún la visión holística de la carrera. La incorporación de TIC ha permitido nuevas formas más interesantes para esta franja de estudiantes- de aprendizaje de la asignatura al ofrecer recursos valiosos (entre los que se puede citar tutoriales multimedia, aulas y laboratorios virtuales) que permiten al docente innovar en el enfoque de enseñanza de determinados contenidos.

Daza Pérez et al. (2009) consideran que el uso de las TIC permite que los alumnos complementen otras formas de aprendizaje utilizadas en la clase, mejoren la comprensión de conceptos difíciles o imposibles de observar a simple vista o en los laboratorios.

La enseñanza en general y la química en particular avanza hacia un modelo que se aleja cada vez más de la clase magistral como base de la instrucción, en la cual la figura del profesor es el centro del sistema, y se dirige hacia un modelo que fomenta la participación activa del alumno como medio fundamental del aprendizaje (Mondeja y Zumalacárregui, 2009).

En ese sentido, Sanabria y Hernández (2011, p. 274) consideran que "la reforma de la enseñanza universitaria requiere innovar el modelo didáctico de docencia universitaria caracterizado por una enseñanza expositiva y un aprendizaje receptivo, lo que implica una renovación de los procesos, estrategias y metodologías de enseñanza". Los autores señalan además que "el alumnado y el proceso de aprendizaje recobran una relevancia fundamental, se hacen visibles en el modelo pedagógico que se apoya más en el trabajo autónomo del alumnado que en la recepción de apuntes en una clase". "Las universidades e instituciones de Educación Superior deben avanzar hacia procesos de enseñanza bajo formatos múltiples accesibles desde cualquier lugar y en cualquier momento para que tanto la enseñanza como los procesos de aprendizaje se enriquezcan" (Vázquez y Sevillano, 2018, p.114). En este sentido, cobra importancia el concepto de ubicuidad para el proceso de enseñanza aprendizaje. Pensar en ubicuidad es pensar en tecnologías que acerquen al estudiante a la información en cualquier momento y lugar. El aprendizaje ubicuo es un nuevo paradigma educativo desde una perspectiva global, donde el espacio físico no es una variable determinante para aprender (García, 2014).

El uso de recursos TIC en instituciones educativas tendría que permitir a los estudiantes el desarrollo de habilidades que faciliten su continua formación y crecimiento intelectual.

Consideramos con Pintó (2011) que las TIC no son adecuadas o inadecuadas "per se". Su uso no avala una mejor enseñanza. Al introducir innovaciones hay que tener en cuenta muchas variables para optimizar el aprendizaje de los alumnos. La forma de utilizar una herramienta, y no su simple uso, es lo que da lugar a cambios en los resultados obtenidos. Las imágenes pueden ayudar a los estudiantes a entender los hechos y las ideas en el contexto de un marco conceptual, siempre que los profesores ayuden a interpretar la información que transmiten las imágenes.

Entre las posibilidades que ofrecen las TIC se encuentran los videos educativos, un valioso recurso que puede ser utilizado dentro y fuera del aula. Si reconocemos que una imagen es fuente de conocimiento, el video resulta un excelente recurso didáctico. El video es uno de los medios didácticos que, adecuadamente empleado, sirve para facilitar el proceso de enseñanza y aprendizaje de determinados contenidos. Según Cabero (2007, p. 136)

la concepción del video como instrumento de conocimiento vendrá de asumirlo como un elemento de trabajo del grupo-clase, a través del cual se persigue que el alumno deje de ser 
sólo un receptor de códigos verbo icónicos para convertirse en emisor de mensajes didácticos...el video se contempla como medio de obtención de información mediante la grabación de experiencias, situaciones, conductas....su uso se refiere a grabaciones totalmente planificadas necesitando por tanto de un diseño, de la búsqueda de información, de un guion....

Aún siguen vigentes las ventajas propuestas por Cebrián de la Serna (1994) respecto a los tres elementos básicos presentes en los videos didácticos: la interactividad con el sistema y el usuario (play, review, capacidad de pausa, repetición de lo visto), los sistemas de símbolos que utiliza (imagen, animación, gráfico, música) y el mensaje, (formas que puede estar presentado y los contenidos).

Existe una fuerte correlación entre las formas en que se usan las TIC y los resultados que se obtienen; y el componente crucial es el profesor y su enfoque pedagógico (BECTA, citado en Caamaño, 2011b). Todo cambio en la práctica docente requiere de tiempo. El mejor rendimiento se consigue cuando se utiliza una TIC que ha estado muy experimentada por el profesor, y cuando se utiliza de manera planificada, estructurada e integrada en las actividades curriculares y no de forma esporádica y descontextualizada (Webb, citado en Caamaño, 2011b). Hay suficientes evidencias de que los alumnos mejoran en la comprensión de conceptos, en la habilidad para resolver problemas y en su capacidad de razonar y explicar científicamente gracias al uso de simulaciones y modelizaciones.

Respecto a la enseñanza y aprendizaje de la química, coincidimos con Izquierdo (2011) en cuanto a que "saber química es ver la química en el mundo, es compartir unas preguntas que no tienen una respuesta definitiva y es saber actuar de una manera determinada..." (Caamaño 2011a, p. 54).

El lenguaje científico es un lenguaje de especialidad que se caracteriza por un vocabulario específico, que se aprende paralelamente al aprendizaje de cada una de las ciencias. La ciencia utiliza un lenguaje simbólico por medio del cual representamos las magnitudes y las unidades en las que las medimos; fórmulas, ecuaciones químicas y símbolos para representar advertencias de riesgo o de seguridad de los productos químicos. En las últimas décadas, la terminología científica ha adquirido un creciente interés por la gran cantidad de vocablos nuevos que se han tenido que crear debido al vertiginoso avance de las ciencias, la tecnología y la técnica, pero también por la importancia que el lenguaje tiene en la enseñanza y el aprendizaje de las ciencias (Caamaño, 2011c).

En las asignaturas químicas el alumnado dispone, generalmente, de dos guías impresas: una de ellas, con los enunciados de ejercicios y problemas a resolver; y la otra, con las actividades de laboratorio que se desarrollarán durante el cursado. Ambas actividades, seminarios de problemas y clases prácticas de laboratorio, se organizan y desarrollan de acuerdo a un cronograma pre establecido.

Uno de los objetivos de la asignatura Química General es conocer y comprender el cambio químico, el que se representa mediante ecuaciones químicas. Escribir correctamente una ecuación química implica reconocer qué sustancias llamadas reactivos se combinan para obtener un determinado producto o productos; además es necesario saber escribir las fórmulas químicas respectivas.

Este proceso complejo de ruptura de enlaces químicos en los reactivos para formar nuevos enlaces en los productos requiere cierto nivel de abstracción que no siempre tiene el alumno al inicio del dictado de la asignatura, que es el momento en que se desarrollan estos contenidos. Escribir una ecuación química implica, asimismo, aplicar reglas de balanceo de acuerdo con el Principio de Conservación de la Masa y utilizar tres niveles de pensamiento (Triángulo de Johnstone): macroscópico, microscópico y simbólico. En una ecuación química tienen significado propio los coeficientes numéricos delante de las fórmulas químicas, que indican cantidad de moles, y los subíndices numéricos que son parte de una fórmula química e indican la proporción en que se combinan los átomos por cada mol de sustancia. El $\mathrm{mol}$ es la unidad de cantidad de sustancia en el SI (Sistema Internacional de Unidades) y comprender su definición y aplicación en cálculos referidos a aspectos cuantitativos de una reacción química es uno de los obstáculos más frecuentes en el aprendizaje de la estequiometría de ecuaciones químicas.

En ese sentido, Flor Reyes y Garritz, Llorens, Pozo y Gómez, (citados en Martínez y De Longhi, 2011) afirman que "los estudiantes tienen problemas, por ejemplo, en diferenciar subíndices de coeficientes, en relacionar la proporcionalidad en la combinación de partículas con las proporciones de combinación de masas; también suelen establecer falsas reglas de conservación de moles en lugar de conservación de átomos". 
Caamaño e Irazoque (2011, p. 55) dan una serie de recomendaciones respecto al uso adecuado del lenguaje y la terminología

ser exigentes con los alumnos en el uso de los términos y símbolos adecuados. En particular, es importante que en los problemas de Química que implican cálculos, los estudiantes usen símbolos para designar las magnitudes que calculan y para escribir las ecuaciones que permiten calcular las magnitudes...

Sugieren, además, "estar atentos a las publicaciones periódicas de la IUPAC sobre normas de formulación y nomenclatura de las especies químicas" (Caamaño, 2011c, p.171).

\subsection{Objetivos}

- Indagar el efecto de la implementación de videos educativos en la comprensión de los temas Nomenclatura Química, Ecuaciones Químicas y Estequiometria en la Asignatura Química General.

- Analizar la posible diferencia estadísticamente significativa entre los resultados obtenidos, en la evaluación de dichos temas, por la cohorte que contó con apoyo del recurso y la que no contó.

\section{METODOLOGIA}

La experiencia se realizó con 260 alumnos (ingresantes y recursantes) que cursaron Química General para carreras de Ingeniería en la FaCENA (UNNE) en las cohortes 2014 y 2015. Esta última contó con la incorporación de videos sobre los tópicos ya señalados.

El alumnado dispone, además, de una guía de actividades para resolver en clase y se ofrece un horario de consulta (de asistencia no obligatoria) para atender dudas sobre la resolución de ejercicios de práctica y estudio independiente. Para esas horas de estudio los alumnos cuentan con el material videográfico editado.

Los videos fueron grabados con una tableta; las imágenes enfocan la hoja en la que la docente resuelve los distintos ejercicios, y con voz en off, pausada y clara, guía el razonamiento y un posible algoritmo de resolución según la situación planteada. Estos fueron editados y publicados en el sitio de acceso libre YouTube (http://bit.ly/2VPu6va), en el canal de la profesora, autora de los vídeos y titular de la Asignatura. La dirección del sitio web se proporciona a los estudiantes en la clase presencial.

Para el tema "formulación y nomenclatura química inorgánica" fueron editados 11 videos referidos a las diferentes familias de compuestos inorgánicos. Se focalizaron en el mecanismo de escritura de fórmulas químicas por compensación de los números de oxidación de los elementos que la integran, teniendo en cuenta el orden de electronegatividades y el uso de las normas IUPAC para la nomenclatura (Peterson, 2012).

Para el tema "ecuaciones químicas" se editaron y publicaron 7 videos. En cada uno se explica y se muestra el procedimiento a seguir para la escritura de ecuaciones químicas de diferentes compuestos inorgánicos. Se señala la importancia de tener en cuenta el nombre del compuesto a obtener para identificar los reactivos a combinar y estados de oxidación adecuados en función de los sufijos empleados.

Para el tema "cálculos estequiométricos basados en formulas químicas" fueron diseñados y editados 4 videos cuyos guiones contienen explicaciones claras, ordenadas y precisas de la resolución de situaciones de complejidad creciente, resaltando la importancia de las unidades en el cálculo de masas molares, uso de factores de conversión adecuados, conversión de masa (en gramos) a número de moles y viceversa y aplicación del concepto de volumen molar. A manera introductoria se presenta un ejemplo de fórmula química y se analiza toda la información que ella involucra respecto a su composición, tipo de elementos que la integran, número de moles, número de átomos, masa y el volumen en condiciones normales de presión y temperatura (CNPT), cuando de un gas se trata.

Como instrumento de recolección de datos se utilizaron las respuestas dadas al cuestionario del primer examen parcial de Química General de los años 2014 y 2015. El cuestionario consta de catorce ejercicios a resolver, agrupados en tres bloques temáticos.

El primer bloque corresponde a ocho ejercicios sobre formulación y nomenclatura; en cuatro de ellos se les da la fórmula del compuesto y se les pide que lo nombren de acuerdo a normas IUPAC; en los cuatro restantes, dado el nombre del compuesto deben escribir la fórmula química. El segundo bloque corresponde a tres ejercicios de ecuaciones químicas, donde se solicita la escritura de la ecuación química correspondiente a un óxido básico o un óxido ácido; un hidróxido o un hidrácido y una sal (binaria u oxosal). El tercer bloque corresponde a 
tres ejercicios de estequiometria en los que se solicita el cálculo del número de moles a partir de una determinada masa de compuesto (masa/mol) y viceversa (mol/masa); el volumen que ocupará un determinado número de moles o número de moléculas de una sustancia gaseosa o el número de moléculas/átomos correspondientes a un determinado volumen de gas en CNPT.

\section{RESULTADOS Y DISCUSIÓN}

En la figura 1 se presentan los resultados obtenidos (en porcentajes) para ambas cohortes y para los tres temas evaluados.

Se observa que en la cohorte 2014 (sin disponibilidad de videos) en el tema "Formulación y Nomenclatura" las respuestas "incorrectas" y "no contesta" superan en $24 \%$ a las "correctas", mientras que en la cohorte 2015 (con apoyo de videos) esta diferencia es solo de $4 \%$.

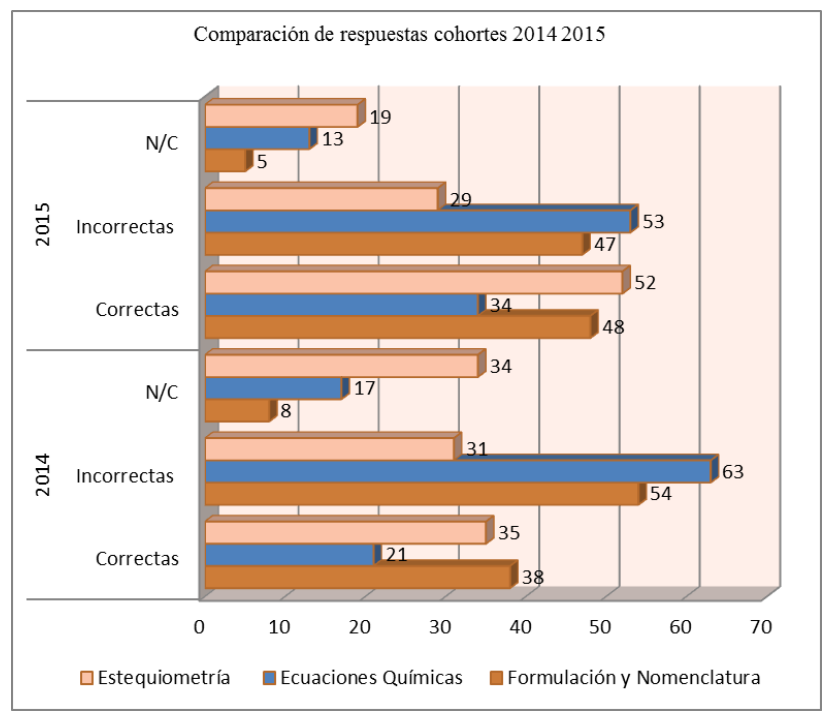

Figura 1. Comparación de respuestas cohortes 2014 y 2015.

En el tema "Ecuaciones Químicas", haciendo el mismo análisis, en 2014 hay 59\% a favor de respuestas "incorrectas" y "no contesta" y en la cohorte 2015 se reduce a $32 \%$ esta diferencia. En el tema "Estequiometria" para la cohorte 2014 las respuestas "incorrecta" y "no contesta" superan 30\% a las correctas y para la cohorte 2015 se invierte la relación, ya que las respuestas correctas superan en $4 \%$ al total de respuestas "incorrectas" y "no contesta". Para ambas cohortes el mayor porcentaje de respuestas incorrectas se obtiene en el tema "Ecuaciones Químicas".

De la comparación de resultados obtenidos por ambas cohortes surge, además, que en el tema
"Formulación y Nomenclatura" las respuestas "correctas" de la cohorte 2015 superan un 10\% a las de la misma categoría en 2014. Para las categorías "incorrectas" y "no contesta" se obtiene una disminución de $10 \%$ en la cohorte que contó con el recurso del video.

Para el tema "Ecuaciones Químicas", la cohorte 2015 supera en $13 \%$ en las respuestas "correctas", mientras que en las "incorrectas" y "no contesta" disminuye 14\%. Para el tema "Estequiometria" se obtiene la mayor diferencia en respuestas "correctas" en la cohorte 2015 (supera en 17\%) e idéntico resultado en cuanto a la disminución de respuestas "incorrectas" y "no contesta".

Luego del análisis descriptivo realizado, se aplicó la prueba "t de Student" para asegurarnos que la diferencia encontrada entre los dos grupos sea significativa. La prueba $\mathrm{t}$ de Student permite evaluar si las medias de dos muestras difieren entre sí de manera significativa. Para ello es necesario otorgar un puntaje a las respuestas realizadas por cada alumno para obtener el valor o calificación que obtuvo cada uno y tomar esta medida como variable de estudio.

Se agruparon los ejercicios del parcial según los tres bloques temáticos y se asignó 1 a cada respuesta correcta y 0 a la respuesta incorrecta o no realizada. De esta manera el bloque de "formulación y nomenclatura" (tabla 1) contenía 8 ítems, entonces los puntajes posibles varían de 0 a 8 , en forma entera; los bloques de "ecuaciones químicas" (tabla 2) y "estequiometria" (tabla 3) contenían 3 ítems cada uno, por lo que los puntajes, varían de 0 a 3 en forma entera.

La cantidad de parciales analizados en cada cohorte -2014 grupo testeo y 2015 grupo experimental- fue 260 .

Para realizar el cálculo de t de Student se definen la hipótesis nula, $\mathrm{H}_{0}$ : No existe diferencia significativa entre los grupos experimental y testeo; la hipótesis alternativa, $\mathrm{H}_{1}$ : existe diferencia significativa entre ambos grupos y el nivel de significación: $\alpha=0,05$.

Realizado el cálculo se tienen los siguientes valores:

Tabla 1. Formulación y nomenclatura

\begin{tabular}{lcccc}
\hline \multicolumn{1}{c}{ Grupo } & $\begin{array}{c}\text { Tamaño } \\
(\mathrm{N})\end{array}$ & $\begin{array}{c}\text { Media } \\
(\overline{\boldsymbol{X}})\end{array}$ & $\begin{array}{c}\text { Varianza } \\
(\mathrm{s})\end{array}$ & $\begin{array}{c}\mathrm{t} \\
\text { Student }\end{array}$ \\
\hline Testeo, 2014 & 260 & 3,49 & 1480,72 & \\
$\begin{array}{l}\text { Experimental, } \\
2015\end{array}$ & 260 & 4,14 & 1632,23 & 2,981 \\
\hline
\end{tabular}

Tabla 2. Ecuaciones químicas 


\begin{tabular}{llccc}
\hline \multicolumn{1}{c}{ Grupo } & $\begin{array}{c}\text { Tamaño } \\
(\mathrm{N})\end{array}$ & $\begin{array}{c}\text { Media } \\
(\overline{\boldsymbol{X}})\end{array}$ & $\begin{array}{c}\text { Varianza } \\
(\mathrm{s})\end{array}$ & $\begin{array}{c}\mathrm{t} \\
\text { Student }\end{array}$ \\
\hline $\begin{array}{l}\text { Testeo, 2014 } \\
\text { Experimental, } \\
2015\end{array}$ & 260 & 0,77 & 280,85 & \\
& 260 & 1,05 & 354,34 & 2,905 \\
\hline
\end{tabular}


Tabla 3. Estequiometria

\begin{tabular}{llccc}
\hline \multicolumn{1}{c}{ Grupo } & $\begin{array}{c}\text { Tamaño } \\
(\mathrm{N})\end{array}$ & $\begin{array}{c}\text { Media } \\
(\overline{\boldsymbol{X}})\end{array}$ & $\begin{array}{c}\text { Varianza } \\
(\mathrm{s})\end{array}$ & $\begin{array}{c}\mathrm{t} \\
\text { Student }\end{array}$ \\
\hline $\begin{array}{l}\text { Testeo, 2014 } \\
\begin{array}{l}\text { Experimental, } \\
200\end{array}\end{array}$ & 260 & 1,87 & 301,57 & \\
2015 & & 391,94 & 5,741 \\
\hline
\end{tabular}

El valor crítico para 518 grados de libertad es: $-1,965<\mathrm{t}<1,965$.

Los valores de $t$ calculados que se muestran en los cuadros anteriores son mayores al valor crítico 1,965 , por lo que se rechaza la hipótesis nula. Esto quiere decir que, en los tres bloques temáticos, la diferencia encontrada entre los grupos es significativa. El cálculo estadístico permite afirmar que el uso del video como recurso es la variable que está provocando la diferencia de tener mejores resultados en la cohorte 2015.

La mayor diferencia entre ambos grupos se tiene para el tema estequiometria y la menor para el tema ecuaciones químicas.

\section{CONCLUSIONES}

Consideramos actuales algunas de las dificultades citadas por Pozo y Gómez (1998) en el aprendizaje de la química:

- Indiferenciación entre cambio físico y cambio químico.

- Atribución de propiedades macroscópicas a átomos y moléculas.

- Dificultades para comprender y utilizar el concepto de cantidad de sustancia.

- Dificultades para establecer las relaciones cuantitativas entre masas, cantidades de sustancia, número de átomos, etc.

- Dificultades para interpretar el significado de una ecuación química ajustada.

Estas dificultades, y otras que podrían completar la lista, según los autores vendrían determinadas por la forma en que el alumno organiza sus conocimientos a partir de sus teorías implícitas sobre la materia.

De acuerdo con los resultados obtenidos, con diferencias estadísticamente significativas entre ambas cohortes, consideramos que el uso de videos como apoyo a la enseñanza de los contenidos analizados, es un recurso eficaz para reducir las dificultades de aprendizaje de estos. Sin embargo, para "ecuaciones químicas" (tema en el que se obtuvo la menor diferencia estadísticamente significativa) se deberá analizar y focalizar la atención para identificar los obstáculos que están impidiendo su comprensión.

La implementación de esta estrategia innovadora evidenció la predilección del alumnado por la aplicación de los videos como recurso de aprendizaje. Esto nos anima a continuar con la tarea de selección de temas y edición de material videográfico a fin de favorecer el proceso de metacognición a través de la observación repetida de los mismos, personalizando los tiempos de aprendizaje.

Estos buenos resultados también llevan a plantearnos la posibilidad de implementar en próximos años la metodología de aula invertida para algunos contenidos del programa de la asignatura, recurriendo para ello a videos elaborados por la Cátedra. En este modelo, el docente distribuye los materiales didácticos como las lecturas de artículos o los videos cortos a los estudiantes antes de la sesión presencial. Posteriormente, en el salón de clases, se promueve el aprendizaje activo y cooperativo por medio del estudio de casos y debates (Salas-Rueda y Lugo-García, 2019).

Como docentes que hace algunos años venimos apostando por el uso de videos como apoyo y complemento de las clases presenciales, podemos reconocerlos como una de las causas del mejor rendimiento académico obtenido. Somos conscientes de que, a partir de la producción, diseño y edición de los mismos, se optimizó la comprensión de los temas, al no depender de las estrategias que utiliza el profesor que le fue asignado a su comisión de trabajo. Todos cuentan con la misma explicación de un docente, para abordar los diferentes ejercicios o situaciones problemáticas.

Como docentes con experiencia, y en coincidencia con Mellado (2011), la reserva de conocimiento didáctico de la disciplina nos permite en función de las dificultades de aprendizaje detectadas en algunos temas de Química Generalcontinuar innovando en las estrategias utilizadas para motivar a nuestros estudiantes, mejorar la comprensión de conceptos difíciles y la habilidad para resolver ejercicios y problemas.

\section{REFERENCIAS}

Caamaño, A. (Coord.) (2011a). Física y Química. Complementos de formación disciplinar. Vol. I. Barcelona, España: Graó.

Caamaño, A. (Coord.) (2011b). Didáctica de la Física y la Química. Vol. II. Barcelona, España: Graó.

Caamaño A. (Coord.) (2011c). Física y Química. Investigación, innovación y buenas prácticas. Vol. III. Barcelona, España: Graó. 
Caamaño, A.; Irazoque, G. (2009). La enseñanza y el aprendizaje de la terminología química: magnitudes y símbolos. Educacio Química EduQ,3, 46-55. doi:10.2436/20.2003.02.24

Cabero Almenara, J. (2007). El video en la enseñanza y formación. En J. Cabero (coord.), Nuevas tecnologías aplicadas a la educación (pp.129-149). Madrid: McGraw-Hill.

Cacheiro González, M.L. (2011). Recursos educativos TIC de información, colaboración y aprendizaje. Pixel-Bit. Revista de Medios y Educación, 39, 69-81.

Cebrián de la Serna, M. (1994). Los videos didácticos: claves para su producción y evaluación. Pixel-Bit. Revista de Medios y Educación, 1, 31-44.

Daza Pérez, E. P., Gras-Marti, A., Gras-Velázquez, À., Guevara, N. G., Togasi, A. G., Joyce, A., ... Santos, J. (2009). Experiencias de enseñanza de la química con el apoyo de las TIC. Educación Química, 20(3), 320-329. doi:10.1016/S0187-893X(18)30032-6

García, G. A. (2014). A comparative study of computer and mobile phone-mediated collaboration: the case of university students in Japan. Mobile Learning Applications in Higher Education. RUSC. Universities and Knowledge Society Journal, 11(1), 222-237. doi:10.7238/rusc.v11i1.1898

Izquierdo, M. (2011). Historia de la Química y enseñanza de la Química. En A. Caamaño (coord.), Física y Química. Complementos de formación disciplinar. Vol. I (pp.5366). Barcelona, España: Graó.

Martínez, M.S., y De Longhi, A. (2011). Identificación de las dificultades en la interpretación de enunciados de problemas en química aplicada. E-book. Córdoba: Universidad Nacional de Córdoba. Disponible en https://rdu.unc.edu.ar/handle/11086/2150.

Mellado, V. (2011). Formación del profesorado de ciencias y buenas prácticas: el lugar de la innovación y la investigación didáctica. En A. Caamaño (Coord.), Física y Química. Investigación, innovación y buenas prácticas, Vol. III, pp.11-26. Barcelona, España: Graó.

Mondeja González, D., y Zumalacárregui de Cárdenas, B. (2009). Química virtual en la enseñanza de las ingenierías de perfil no químico. Pedagogía Universitaria, 14(1), 9-17.

Morales Capilla, M., Ortiz Colón, A.M., Trujillo Torres, J.M., y Raso Sánchez, F. (2015). Percepción del alumnado universitario acerca del uso e integración de las TIC en el proceso educativo en la Facultad de Educación de Granada. Innoeduca. International Journal of Technology and Educational Innovation. 1(2), 57-68. doi:10.20548/innoeduca.2015.v1i2.1035

Peterson, W. R. (2012). Fundamentos de Nomenclatura Química. Barcelona: Reverté.

Pintó, R. (2011). Las Tecnologías digitales en la enseñanza de la Física y de la Química. En A. Caamaño (Coord), Didáctica de la Física y la Química, Vol. II (pp.172179). Barcelona, España: Graó.

Pozo, J.I. y Gómez Crespo, M.A. (1998). Aprender y Enseñar Ciencias. Madrid, España: Morata.

Salas-Rueda, R.A., y Lugo-García, J. L. (2019). Impacto del aula invertida durante el proceso educativo superior sobre las derivadas considerando la ciencia de datos y el aprendizaje automático. EDMETIC, Revista de Educación Mediática y TIC, 8(1), 147-170. doi:10.21071/edmetic.v8i1.9542
Sanabria Mesa, A. L. y Hernández Jorge, C. M. (2011). Percepción de los estudiantes y profesores sobre el uso de las TIC en los procesos de cambio e innovación en la Enseñanza Superior. Aloma, 29, 273-290.

Vázquez Cano, E. y Sevillano García, M.L. (2018). Uso educativo ubicuo de dispositivos digitales móviles. Un estudio general y comparativo en la Educación Superior de España y Latinoamérica. Journal New Approaches in Educational Research, 7(2), 105-115. doi:10.7821/naer.2018.7.308 